\title{
Long lived acoustic vibrational modes of an embedded nanoparticle
}

\author{
Lucien Saviot ${ }^{1}$ and Daniel B. Murray ${ }^{2}$ \\ ${ }^{1}$ Laboratoire de Recherche sur la Réactivité des Solides, UMR 5613 CNRS - Université de Bourgogne \\ 9 avenue A. Savary, BP 47870 - 21078 Dijon - France* \\ ${ }^{2}$ Department of Physics and Astronomy, Okanagan University College, \\ 3333 College Way, Kelowna, British Columbia, Canada V1V $1 \mathrm{~V}$ 开
}

(Dated: November 15, 2018)

\begin{abstract}
Classical continuum elastic calculations show that the acoustic vibrational modes of an embedded nanoparticle can be lightly damped even when the longitudinal plane wave acoustic impedances $Z_{o}=\rho v_{L}$ of the nanoparticle and the matrix are the same. It is not necessary for the matrix to be less dense or softer than the nanoparticle in order to have long lived vibrational modes. A corrected formula for acoustic impedance is provided for the case of longitudinal spherical waves. Continuum boundary conditions do not always accurately reflect the microscropic nature of the interface between nanoparticle and matrix, and a multi-layer model of the interface reveals the possibility of additional reduction of mode damping.

PACS numbers: $62.20 .-\mathrm{x}, 43.20 . \mathrm{Ks}, 62.25 .+\mathrm{g}$
\end{abstract}

A classical vibrational mode weakly coupled to a cold environment will have an amplitude exponentially decaying with time. The vibrational frequency of the mode may be slightly shifted from what it would have been if the environmental coupling is reduced to zero. When coupling is stronger, the mode loses its individual identity, and it becomes necessary instead to consider normal modes of the entire system.

Nearly spherical clusters of atoms a few nanometers in size (nanoparticles) have mechanical vibrations which can be initiated through sudden thermal excitation with a brief laser pulse. 1] Subsequent evolution of the vibration can be followed by observing the amount of light absorption by a probe pulse following the pump pulse by a controlled time.

It is rarely possible to perform such experiments on isolated nanoparticles. Normally there is mechanical coupling to an environment. This can be (1) a powder of nanoparticles (where adsorption is important) (2) a substrate to which the nanoparticle is attached (3) liquid solution or (4) a solid surrounding matrix, usually glass. Propagation of acoustic waves away from the nanoparticle is often the dominant mechanism for mechanical energy loss. As a result, vibrational modes are damped.

The organization of this paper is as follows: We begin with the current "state of the art" of vibration eigenmode calculation for free and matrix-embedded spheres. Then we focus on a particular material combination which gives rise to very low damping of the fundamental "breathing" mode even though the longitudinal plane wave acoustic impedances in the two materials are the same. We then derive the correct expression for the acoustic impedance for such modes and explain this result. Finally, we extend the calculation to more realistic materials where the sphere-matrix contact is not perfect and to modes having different symetries.

A quantum mechanical analysis of energy loss of a nanoparticle weakly coupled to a substrate heat bath has been done. 2] Otherwise, the nanoparticle has been approximated as a classical isotropic homogeneous linear elastic continuum object in contact with a similarly idealized zero temperature matrix. We consider such a spherical nanoparticle of radius $R_{p}$, density $\rho_{p}$ and speeds of sound $v_{L p}$ and $v_{T p}$ embedded within a macroscopic matrix of density $\rho_{m}$ and speeds of sound $v_{L m}$ and $v_{T m}$. Idealized continuum boundary conditions [3] of continuous displacement and normal stress components have been applied where the nanoparticle contacts the matrix, but these will be reaxamined later on.

"Pseudomodes" of a nanoparticle embedded in a solid or fluid matrix can be found [4] yielding complex valued frequencies $\omega_{q \ell n}^{\mathrm{CFM}}$ of the "complex frequency model" (CFM). $q$ can be torsional (TOR) or spheroidal (SPH). $\ell$ is the angular momentum and $m$ is the $z$-component. $n \geq 0$ is the mode index. $\operatorname{Re}\left(\omega_{q \ell n}^{\mathrm{CFM}}\right)=2 \pi / T$ are shifted from $\omega_{q \ell n}^{\mathrm{FSM}}$ of a free sphere model (FSM) as first calculated in 1882 by Lamb [5] and some new modes appear. [6] $\operatorname{Im}\left(\omega_{q \ell n}^{\mathrm{CFM}}\right)=1 / \tau=\frac{1}{2} \Delta \omega$ where $\tau$ is the damping time and $\Delta \omega$ is the full width at half maximum (FWHM) of a Brillouin or Raman peak. The quality factor of each pseudomode is defined as $Q=\omega / \Delta \omega$. This paper is mainly focused on $(\mathrm{SPH}, \ell=0)$ modes which are the only ones to have been experimentally observed in pumpprobe experiments. These modes are also Raman active.

Interpretation of CFM is not straightforward, since the resulting pseudomodes are not orthonormalizable and blow up exponentially with the radial coordinate. This makes a correspondence to quantum theory unclear. An alternative is to calculate all of the normal modes of a nanoparticle ("core") surrounded by a large spherical matrix ("shell"), imposing an arbitrary condition on the matrix outer surface. 7] The resulting core-shell model (CSM) is conservative and has real valued mode frequencies. [6] However, there is a continuum of normal 
modes in the macroscopic limit that the matrix radius is infinite. Note that highly damped "matrix modes" [6] are not considered in this work.

The connection between the continuum of CSM modes and the discrete spectra of CFM pseudomodes is clearly seen by making a plot of the mean squared displacement within the nanoparticle $\left\langle u^{2}\right\rangle_{p}$ as a function of frequency for CSM modes. [6] The resulting peaks correspond closely to $\operatorname{Re}\left(\omega_{q \ell n}^{\mathrm{CFM}}\right)$ and the FWHM of the CSM peaks corresponds closely to $2 \operatorname{Im}\left(\omega_{q \ell n}^{\mathrm{CFM}}\right)$.

Even without applying the complexities of CFM or CSM, the qualitative effect of the matrix in shifting and broadening FSM frequencies can often be correctly anticipated by comparing the longitudinal plane wave acoustic impedances (LPWAI) $Z_{o p}=\rho_{p} v_{L p}$ and $Z_{o m}=\rho_{m} v_{L m}$.

The "rule of thumb" is that nanoparticle vibrational modes will be lightly damped when the LPWAI's satisfy $Z_{o p} \gg Z_{o m}$ or $Z_{o p} \ll Z_{o m}$. Otherwise, similar LPWAI's of the nanoparticle and matrix allow vibrational energy to freely flow out, resulting in strong damping.

Here we report dramatic exceptions to this. It is possible for a nanoparticle to have lightly damped normal modes without LPWAI's $Z_{o m}$ and $Z_{o p}$ being very different, with the extreme possibility of $\tau$ being large even if $Z_{o m}=Z_{o p}$. Furthermore, lightly damped nanoparticle frequencies can differ greatly from $\omega_{q \ell n}^{\mathrm{FSM}}$. These possibilities have remained obscure up to now because experiments were limited primarily to situations in which the LPWAI's satisfied $Z_{o p}>Z_{o m}$ for which FSM works extremely well. Furthermore, nanoparticle size variation within a sample creates extrinsic broadening of the modes which can sometimes exceed intrinsic broadening.

An example will illustrate when such unexpected results can occur: a gold nanoparticle embedded in a diamond matrix. We use the elastic constants of bulk fcc gold at $300 \mathrm{~K}[\underline{8}]$ and use directional averaging $[9]$ to obtain longitudinal and transverse speeds of sound of $3330 \mathrm{~m} / \mathrm{s}$ and $1250 \mathrm{~m} / \mathrm{s}$ respectively. We use elastic constants for bulk crystalline diamond at $300 \mathrm{~K} 10$ and similarly obtain speeds of sound $18190 \mathrm{~m} / \mathrm{s}$ and $12350 \mathrm{~m} / \mathrm{s}$. The resulting LPWAI's are identical: $Z_{o p} \approx$ $Z_{\text {om }} \approx 6.4 \cdot 10^{7} \mathrm{~kg} \mathrm{~m}^{-2} \mathrm{~s}^{-1}$. In the absence of any apparent impedance mismatch, the "rule of thumb" says that the vibrational modes of the nanoparticle would become so broadened by the presence of the matrix as to be unrecognizable.

Figure 1 shows something completely different. $\left\langle u^{2}\right\rangle_{p}$ for CSM (SPH, $\ell=0)$ modes is plotted versus frequency. For comparison, $\omega_{q \ell n}^{\mathrm{CFM}}$ are plotted showing $\operatorname{Re}(\omega)$ as a vertical arrow with horizontal arrows to indicate $\operatorname{Im}(\omega)$ which is half the FWHM. The resulting CSM peaks and $\operatorname{Re}\left(\omega_{q \ell n}^{\mathrm{CFM}}\right)$ match very closely. The gold nanoparticle has several lightly damped modes of vibration.

Figure 2 shows how misleading naive comparison of LPWAI's can be. It was obtained by varying both $\rho_{m}$ and $v_{L m}$ while keeping $Z_{o m}=Z_{o p}$. Mode broadening is large

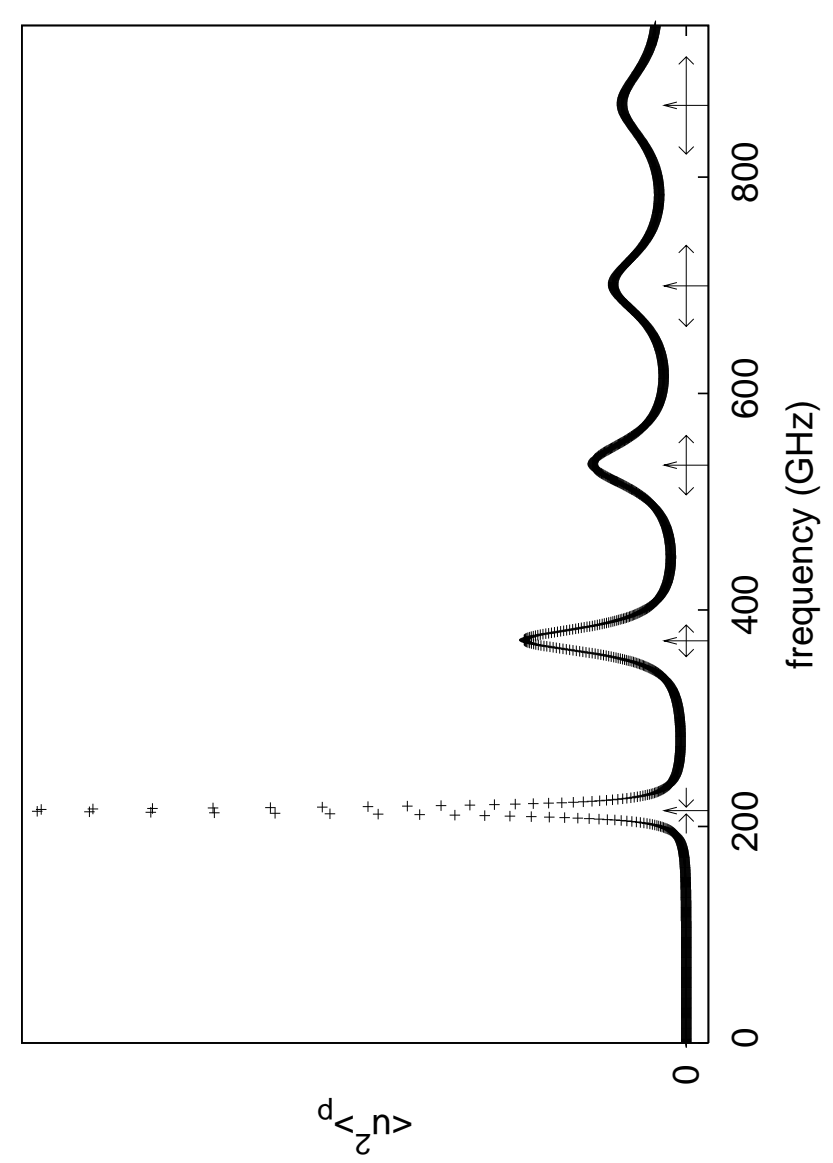

FIG. 1: Mean square displacement within the nanoparticle interior of CSM (SPH, $\ell=0)$ vibrational modes for $\mathrm{Au}$ (radius $R_{p}=10 \mathrm{~nm}$ ) in diamond (radius $R_{m}=2000 \cdot R_{p}$ ). Arrows indicate positions and FWHM obtained with the CFM.

only when $v_{L m}<v_{L p}$. Note that for a gold nanoparticle in diamond, $v_{T m} / v_{L m} \approx 0.68$ and $\rho_{m} / \rho_{p} \approx 0.18$. For low matrix mass density, the frequency approaches that of the bound sphere model (BSM), $\omega_{\mathrm{SPH}}^{\mathrm{BSM} 0}$, which is for a sphere with zero displacement boundary conditions.

For a purely longitudinal spherical wave in an isotropic elastic medium, the acoustic impedance is $Z=-\sigma_{r r} / v_{r}$ where stress component $\sigma_{r r}=2 \rho v_{T}^{2} e_{r r}+\rho\left(v_{L}^{2}-\right.$ $\left.2 v_{T}^{2}\right)\left(e_{r r}+e_{\theta \theta}+e_{\phi \phi}\right)$ and velocity $v_{r}=\partial u_{r} / \partial t$. For this case strain $e_{r r}=\partial u_{r} / \partial r$ and $e_{\theta \theta}=e_{\phi \phi}=u_{r} / r$. The radial displacement is $u_{r}=e^{-i \omega t}(d / d r)\left(e^{i k_{L} r} / r\right)$ where $\omega=v_{L} k_{L}$. The resulting expression for impedance is

$$
Z(s)=\frac{\rho\left(v_{L}^{2}+4 v_{T}^{2}\left(i s-s^{2}\right)\right)}{v_{L}(1+i s)}
$$

where $s=1 /\left(k_{L} r\right)$. This can only be applied to the case of $(\mathrm{SPH}, \ell=0)$ modes. In the plane wave limit (large $r) Z \rightarrow \rho v_{L}$. For a fluid medium, $v_{T}=0$ so that $Z=\rho v_{L} /(1+i s)$, a result familiar for sound waves 11. Another interesting case is Poisson ratio $\nu=\frac{1}{3}$ (approx- 


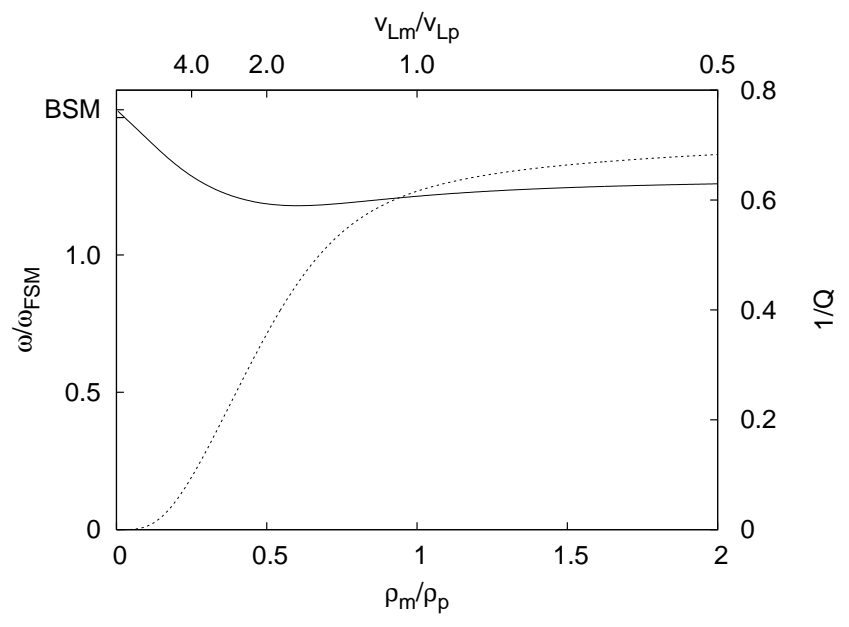

FIG. 2: Normalized CFM frequency (line, left axis) and $1 / Q=\Delta \omega / \omega$ (dots, right axis) of the fundamental (SPH, $\ell=$ $0)$ mode along $Z_{o m} / Z_{o p}=\rho_{m} v_{L m} / \rho_{p} v_{L p}=1$ where $v_{T p} / v_{L p}=0.375$ (gold) and $v_{T m} / v_{L m}=0.6 . \Delta \omega$ is the full width at half maximum.

imately true of many materials) where $v_{T}=\frac{1}{2} v_{L}$ so that $Z=\rho v_{L}$ to linear order in $s$. Note that $v_{T} / v_{L}=\sqrt{\frac{1-2 \nu}{2-2 \nu}}$.

If $s$ is small, then $\operatorname{Re}(Z) \approx \rho v_{L}$ and $\operatorname{Im}(Z) \approx$ $s \rho v_{L}\left(4\left(v_{T} / v_{L}\right)^{2}-1\right)$. Note that if $v_{T}=\frac{1}{2} v_{L}$ then $\operatorname{Im}(Z)$ is approximately zero.

Define $\alpha=v_{L m} / v_{L p}$. Let $Z_{p}(1 / \xi)$ and $Z_{m}(\alpha / \xi)$ denote the acoustic impedance evaluated at $R_{p}$ of the nanoparticle and matrix respectively for outgoing waves of dimensionless frequency $\xi=\omega R_{p} / v_{L p}$. Note that for a given pseudomode, these impedances do not depend on $R_{p}$. If $Z_{p}(1 / \xi)=Z_{m}(\alpha / \xi)$ then a longitudinal travelling wave can cross the nanoparticle-matrix interface without any reflection.

Consider $Z_{p}(1 / \xi)$ : for the (SPH, $\left.\ell=0, n\right)$ FSM mode, $s\left(r=R_{p}\right) \sim \frac{1}{(n+1) \pi}$. For the fundamental $(n=0)$ mode $s\left(r=R_{p}\right)$ is not small and $Z_{p}(1 / \xi)$ is complex-valued. But for higher order modes $s$ becomes small so that $Z_{p}(1 / \xi) \rightarrow \rho_{p} v_{L p}$. Thus, it is expected that the acoustic impedance mismatch at the nanoparticle-matrix interface will be different for higher order modes. Figure 1] confirms this. Also, as predicted in a previous paper $[\overline{6}]$, the area under each pseudomode is roughly constant in the $\left\langle u^{2}\right\rangle_{p}$ plot. This is important because it means that less damped modes compensate their lower frequency broadening with a higher mean square displacement and therefore can have similar electron-phonon coupling inside the nanoparticle as more damped modes.

Consider the longitudinal radial $(r)$ velocity within the nanoparticle $(p)$ to be the sum of inward $(i)$ and outward (o) travelling waves:

$$
\begin{aligned}
& v_{r p o}(x)=e^{-i \omega t} A_{p o} \omega e^{i x}\left((1 / x)+\left(i / x^{2}\right)\right) \\
& v_{r p i}(y)=e^{-i \omega t} A_{p i} \omega e^{i y}\left((1 / y)+\left(i / y^{2}\right)\right)
\end{aligned}
$$

where $x=\omega r / v_{L p}$ and $y=-\omega r / v_{L p} . A_{p o}$ and $A_{p i}$ are amplitudes. To avoid singularity at the origin, $A_{p o}=$ $-A_{p i}$. Matching of boundary conditions at the interface at $R_{p}$ corresponds to the equation:

$$
\frac{Z_{p}(1 / \xi)-Z_{m}(\alpha / \xi)}{Z_{p}(-1 / \xi)+Z_{m}(\alpha / \xi)}=\frac{v_{r p i}(-\xi)}{v_{r p o}(\xi)}
$$

The roots of this equation are the complex valued dimensionless frequencies $\xi_{\mathrm{SPH} 0 n}^{\mathrm{CFM}}$. This equation is equivalent to previous solutions [4] of CFM for the $(\mathrm{SPH}, 0)$ case, but clearly illustrates the role of the acoustic impedances in determining the mode frequencies.

Previous studies 12 were restricted to analyzing the situation of a soft, light matrix where $v_{L m}<v_{L p}$ and $\rho_{m}<\rho_{p}$, and found that $\tau$ does not depend on $n$. But we find that in other situations damping can vary dramatically with mode order, as clearly seen in Fig. 1

In the matrix, $s=\alpha / \xi$ can be small if $v_{L m} \ll v_{L p}$. But if $v_{L m} \gg v_{L p}$ then $s$ can be large, and in this limit $Z_{m}(\alpha / \xi) \rightarrow 4 i \rho_{m} v_{T m}^{2} /\left(\omega R_{p}\right)$, and the effect of the matrix is a purely restorative force, as if the matrix is a massless spring. Energy cannot be absorbed by the matrix in this limit, so that $\tau \rightarrow \infty$ when $v_{L p} / v_{L m} \rightarrow 0$.

We have explored the lowest $(\mathrm{SPH}, \ell=0)$ mode (ignoring highly damped "matrix modes" when they are present) in the parameter space of $\rho_{p}, \rho_{m}, v_{L p}, v_{T p}, v_{L m}$ and $v_{T m}$ and found some general patterns. Notably, good impedance matching between nanoparticle and matrix as exhibited in very strong lowest $(\mathrm{SPH}, 0)$ mode damping is an unusual occurence requiring special conditions. Closeness of the LPWAI's alone is not sufficient.

If $\nu_{m} \ll \nu_{p}$ (where $\nu_{m}$ and $\nu_{p}$ are the Poisson ratios of the matrix and particle respectively) then there is never strong damping, but we can have strong damping if $\nu_{m} \gg \nu_{p}$ such as for a liquid matrix. Assuming the Poisson ratios are close, it is usually necessary that $\rho_{m} \geq \rho_{p}$ and $v_{L m} \leq v_{L p}$. Under these conditions, $s$ is small in the matrix and the LPWAI's are close to the true impedances. In addition, if $\nu_{m}$ and $\nu_{p}$ are both close to $\frac{1}{3}$ then strong damping is still possible if $\rho_{m}$ is as little as $0.8 \rho_{p}$. Conversely, light damping is always seen when any of the following conditions is satisfied: (1) $\nu_{p} \gg \nu_{m}$ (2) $\rho_{m} \ll 0.8 \rho_{p}(3) v_{L m} \gg 1.2 v_{L p}$.

As was shown in a previous work, [7] the microscopic details of the interface (e.g. the specific nature of the mechanical connection of the gold crystal lattice to the diamond matrix) can tend to isolate (i.e. decouple) the nanoparticle from the surrounding medium. In particular, continuum-type boundary conditions applied to the interface may not be optimal. [3] One rough-and-ready way to adapt the CFM and CSM models to tentatively take this into account is to introduce an intermediate shell layer (X-layer) of inner radius $R_{p}-d_{X} / 2$, outer radius $R_{p}+d_{X} / 2$, density $\rho_{X}$ and speeds of sound $v_{L X}$ and $v_{T X}$. Depending on the elaboration process, the 
nanoparticle and the surrounding matrix do not necessarily tightly adhere. Different expansion coefficients for example could result in a void in the matrix bigger than the nanoparticle and the nanoparticle being only partially in contact with the matrix. The X-layer is the most elementary way to explore the effect of deviations from continuum boundary conditions. Such calculations are relatively easy to perform using CFM or CSM.

It is important to demonstrate the robustness of CFM results against the microscopic details of the interface. The nature of the interface is unknown, but it can be guessed to be of lower density, poorly-ordered and not strongly-bonded. It is plausible to assume a low density and low elastic constants comparable to water or plastic. If $d_{X}$ is thin, neither $\rho_{X}$ nor $v_{T X}$ have a strong effect. What is important is the elastic constant of the X-layer in terms of $\rho_{X} v_{L X}^{2}$ relative to the thickness of the Xlayer, $d_{X}$. It is convenient to introduce the dimensionless parameter $\sigma_{X}=d_{X} \rho_{p} v_{L p}^{2} /\left(R_{p} \rho_{X} v_{L X}^{2}\right)$. For examples we looked at, the larger $\sigma_{X}$ is, the larger $\tau$ and $Q$ become.

A possible example of this may have been seen for gold nanoparticles embedded in $\mathrm{TiO}_{2}$. This is unrelated to our previous example of $\mathrm{Au}$ in diamond. Experimental observations of nanoparticle "breathing modes" (those with purely radial vibration) for $\mathrm{Au}$ nanoparticles in a $\mathrm{TiO}_{2}$ matrix using the femtosecond pump probe technique 13 . reveal surprisingly light damping. Qian et al. obtained an oscillation frequency of $0.15 \mathrm{THz}\left(5 \mathrm{~cm}^{-1}\right)$ with a damping time of $55 \mathrm{ps}(Q \approx 26)$ for $R_{p}=10 \mathrm{~nm}$. We use $\mathrm{TiO}_{2}$ sound speeds of $8610 \mathrm{~m} / \mathrm{s}$ and $5160 \mathrm{~m} / \mathrm{s}$ and density $4.097 \mathrm{~g} / \mathrm{cm}^{3}$. 14 Our XCFM calculations for very small $\sigma_{X}$ predict $\tau \approx\left(10.7+21.0 \sigma_{X}\right)$ ps or $Q \approx 5.92+11.7 \sigma_{X}$. This $\tau$ provides only an upper bound on the experimental damping time because of dephasing due to particle size variation. The large $\tau$ of $\mathrm{Au}$ in $\mathrm{TiO}_{2}$ could be obtained by a water-like layer between the nanoparticle and the matrix on the order of $0.1 \mathrm{~nm}$ thick. Thus, details of the nanoparticle-matrix interface at the atomic level are crucial in determining the damping of embedded nanoparticle vibrational modes. However, proper first-principles quantitative modelling of the interface requires consideration of detailed features of crystal and electronic properties at the atomic level, in particular the nature of the bonding. 15] For the systems we consider, there is insufficient information about the interface to attempt such a calculation, especially since the matrix is amorphous.

The ability to choose the matrix so as to have nanoparticles with lightly damped vibrational modes is important for several reasons. First, by reducing homogeneous broadening, it allows inhomogeneous broadening effects to stand out clearly. This results in a clearer picture for femtosecond pump-probe experiments where the damping time is longer but also in low frequency Raman experiments where a better separation of scattering lines is possible. Second, as was shown by the pump-pumpprobe experiments in Del Fatti et al. [16] it is possible to control the acoustic motion of silver nanoparticles. With higher $Q$ factor, the first pump pulse would "launch" the motion of every nanoparticles but the next pump pulse could selectively enhanced the vibration amplitude of a smaller subset of the nanoparticles.

Solid matrix-embedded nanoparticles always have $Q$ factor lower than free ones. However, free nanoparticles suffer from many drawbacks. For example, surface adsorption can affect their properties. Also it is often technologically easier to deal with matrix embedded nanoparticles. Where solid embedding is technologically desirable, our results provide guidance about how the matrix can be optimally chosen.

Finally, the sphere radius is irrelevant in this paper ( $Q$ does not depend on $R$ ). So there are some potential applications for other kinds of systems. Such damped vibrational modes of a virus in water may be relevant to its mechanical response to ultrasound. 17.

All of the foregoing discussion applies only to spheroidal vibrations with $\ell=0$. A similar analysis can be carried out for torsional modes, but a separate expression for acoustic impedance is required for each value of $\ell$. It is not apparent how to make the extension to spheroidal modes with $\ell \neq 0$. Calculations made on $\operatorname{CdS}$ spheres embedded in silica in a previous work $[\underline{6}]$ revealed lightly damped fundamental torsional modes which we could not understand at that time. The situation is even more dramatic for gold spheres inside diamond for which the lowest torsional modes have very high quality factors $(Q \sim 900$ for $($ TOR, $\ell=1, n=0), Q \sim 20000$ for $(\mathrm{TOR}, \ell=2, n=0), \ldots)$

* Electronic address: lucien.saviot@u-bourgogne.fr

† Electronic address: dmurray@ouc.bc.ca

[1] C. Voisin, N. Del Fatti, D. Christofilos, and F. Vallée, J. Phys. Chem. B 105, 2264 (2001), and references therein.

[2] K. R. Patton and M. R. Geller, Phys. Rev. B 67, 155418 (2003), cond-mat/0202325.

[3] C. W. Bettenhausen, W. C. Bowie, and M. R. Geller, Phys. Rev. B 68, 035431 (2003), cond-mat/0103066.

[4] V. A. Dubrovskiy and V. S. Morochnik, Earth Physics 17, 494 (1981).

[5] H. Lamb, Proc. London Math. Soc. 13, 189 (1882).

[6] D. B. Murray and L. Saviot, Phys. Rev. B 69, 094305 (2004), cond-mat/0310099.

[7] H. Portalès, L. Saviot, E. Duval, M. Gaudry, E. Cottancin, M. Pellarin, J. Lermé, and M. Broyer, Phys. Rev. B 65, 165422 (2002), cond-mat/0203468.

[8] R. F. S. Hearmon, in The elastic constants of crystals and other anisotropic materials, edited by K. H. Hellwege and A. M. Hellwege (Springer-Verlag, Berlin, 1984), no. III/18 in Landolt-Börstein Tables, pp. 1-154.

[9] L. Saviot, D. B. Murray, and M. del C. Marco de Lucas, Phys. Rev. B 69, 113402 (2004), cond-mat/0307634.

[10] H. J. McSkimin and W. L. Bond, J. Appl. Phys. 43, 2944 (1972). 
[11] L. E. Kinsler, A. R. Frey, A. B. Coppens, and J. V. Sanders, Fundamentals of Acoustics (Wiley, 2000), 4th ed.

[12] C. Voisin, D. Christofilos, N. Del Fatti, and F. Vallée, Physica B 316-317, 89 (2002).

[13] W. Qian, L. Lin, Y. J. Deng, Z. J. Xia, Y. H. Zou, and G. K. L. Wong, J. Appl. Phys. 87, 612 (2000).

[14] W. P. Minnear and R. C. Bradt, J. Am. Ceram. Soc. 60,
458 (1977).

[15] P. Carrier, L. J. Lewis, and M. W. C. Dharma-wardana, Phys. Rev. B 65, 165339 (2002).

[16] N. Del Fatti, C. Voisin, D. Christofilos, F. Vallée, and C. Flytzanis, J. Phys. Chem. A 104, 4321 (2000).

[17] L. Saviot, D. B. Murray, A. Mermet, and E. Duval, Phys. Rev. E 69, 23901 (2004), cond-mat/0307112. 